\title{
A Correlative Study on Bone Marrow Angiogenesis with Bone Marrow Fibrosis and Splenomegaly
}

\author{
Anbu Lenin Kulandaivel ${ }^{1 *}$ and Kumudhini Priya Gunasekaran ${ }^{2}$ \\ 'Department of Pathology, Vinayaka Mission's Kirupananda Variyar Medical College And Hospital, Salem Tamil Nadu, India. \\ ${ }^{2}$ Department of Pathology, Karuna Medical College, Vilayodi-Chittur, Palakkad, Kerala, India.
}

\begin{abstract}
Background: Myelofibrosis is characterised by increased deposition of collagen type I and III in the bone marrow. Fibroplasia is associated with increased blood flow through marrow substance. Fibrosis occurs in variety of haematological and non haematological disorders. The present study was conducted to study the pattern of mean vessel density in various pathological disorders presenting with marrow fibrosis and to correlate it with spleen size.

Methods: This is one year observational study undertaken in Department of pathology, Coimbatore medical college hospital, Coimbatore. A total of 25 cases presented with bone marrow fibrosis were taken and correlative study was done with mean vessel density and splenomegaly.

Result: Of a total 25 Patients, 17 were secondary myelofibrosis and 8were Primary myelofibrosis. Patient from 18 to 80 years were encountered in the study. Total of 13 males and 12 females were included. Among Primary myelofibrosis 6 were males and 2 were females. Most of the cases of secondary myelofibrosis (11 out of 18) were Hematological malignancies which included AML(3), CML(2), CLL(1), MDS(1), NHL(3), MM(1).CD34 Immunostaining showed increased mean vessel density among all the cases of primary myelofibrosis.

Conclusion: Reticulin fibrosis occurs in variety of Hematological, Non Hematological Malignancies and Non neoplastic disorders including Tuberculosis, SLE and post radiotherapy accompanied by various degree of mean vessel density. Increase in MVD is considered as mother event in causing fibrosis and splenomegaly. Hence studying MVD and correlating it with fibrosis and splenomegaly will be helpful in evaluating the disease progression.
\end{abstract}

Keywords: Myelofibrosis, Reticulin Fibrosis, Splenomegaly, CD34, Mean Vessel Density.

\section{Introduction}

Bone Marrow is involved in various Hematological and Non Hematological disorders. Hematological Malignancies includes Acute Leukemias, Chronic Myelproliferative Neoplasms, Chronic lymphocytic leukemias, Myelodysplastic syndromes, and Lymphomas. Non Hematological Disorders includes Metastasis from Various solid Tumours and Infectious disorders like Tuberculosis and Parasitic infections. Bone marrow fibrosis occurs in various Disorders and is not unique to Primary Myelofibrosis. The fibroblastic proliferation in marrow is not an Intrinsic part of the abnormal clonal expansion of hematopoiesis ${ }^{[1]}$. Angiogenesis is integral to solid tumor growth and metastasis ${ }^{[2]}$. In Hematological diseases, Bone marrow is a primary site of disease activity and a readily accessible tissue for the investigation of angiogenesis. Under normal conditions, the human bone marrow is supplied by a small number of blood vessels. The concentration of these vessels or bone marrow microvessel density is increased in various haematological disorders including acute lymphoid leukemia ${ }^{[3]}$,acute myeloid leukemia ${ }^{[4]}$,Myelodysplastic syndrome ${ }^{[5]}$,Chronic myeloid leukemia $^{[6]}$ and plasma cell disorder ${ }^{[7]}$.Furthermore , Increase in microvessel density has been correlated with unfavourable prognosis in multiple myeloma. Angiogenesis plays a critical role in the development and progression of neoplasia, fibrosis and splenomegaly and Hence evaluation of microvessel density and its correlation with fibrosis and splenomegaly is of prognostic importance.

\section{Materials and Methods}

This was a Prospective Study Conducted in the Department Of Pathology, Coimbatore Medical College Hospital,Coimbatore over a period of One year . During the period, 25cases which showed bone marrow fibrosis in routine hematoxylin and eosin stain were selected for the study. A preforma was used to register various informations about the patients including Name, Age, Sex and Clinical profiles including Presenting Symptoms, Past history and Treatment history. Hematological Profiles including Hemoglobin, Total Leucocyte Count and Platelet Count were studied using Automatic Analyser Sysmex KX-21. Morphological abnormalities of Red Blood Cells, White Blood Cells and Platelets were studied using peripheral smears stained with leishman stain. 
Bone Marrow Biopsy: Bone Marrow Biopsies received were fixed in $10 \%$ Neutral Buffered Formalin and Decalcified in 5\% Nitric acid. Subsequently all were routinely processed, Embedded and 3-4 um Sections were made and stained with Hematoxylin and Eosin (H\&E) Stain, Reticulin Stain. All the Slides were Systematically Studied by two pathologists and Details including Architecture, Cellularity, Dysplastic Megakaryocytes, Megakaryocyte clumping, Fibrosis, Osteosclerosis, Caseaous Necrosis, Granulomas, Fungal colonies, Parasites, Lymphomatous Infiltrate and Metastatic Deposit were Recoreded. Reticulin Fibrosis was graded using modified Bauermeister scale. They were given the score of $0-4$.

Grade0: No reticulin fibres.

Grade1: Occasional fine individual fibres and foci of a fine fibre network.

Grade2: Fine fibre network throughout most of the section. No coarse fibres seen.

Grade3: Diffuse fibre network with scattered thick coarse fibres but no mature collagen.

Grade4: Diffuse often coarse fibre network with areas of collagenisation (positive trichrome staining).

Tumour Angiogenesis: Initially during the study period many antibodies including CD31, Factor VIII, along with CD34 were tried. Finally, CD34 was selected as Antibody of choice. The slides which were stained for CD34 were graded for microvessel density (Neoangiogenesis). The grading was done using visual microvessel density grading system which was introduced by Weidner et al ${ }^{[8]}$ in solid tumours and adopted by Mesa et $\mathrm{al}^{[9]}$. Each of the study slide was first scanned at 100x magnification. Three areas with abundant microvessels were selected and defined as hot spots. The number of microvessels in each of these hot spots was then determined at 400x magnification. The final MVD number [microvessels per high power(400x) field] was assigned by taking the average of the three separate visual counts. During the process of grading MVD, large vessels and vessels in the periosteum or bone and open sinusoids were excluded. Areas of staining with no discrete breaks were counted as single vessel and presence of lumen was not required.

The results obtained were graded as follows by comparing with age matched controls. After grading, the results were compared among themselves and correlated with bone marrow fibrosis(Reticulin fibrosis) and splenomegaly.

MVG I : Slightly Increased.

MVG II : Easy to find and Definitely Increased from normal.
MVG III : Abundant vessels.

MVG IV : Markedly Increased.

Spleen Size: Spleen size of the cases were obtained using ultrasonographic examination and were divided into three categories as mild, moderate and severe. The results were correlated with mean vascular density (MVD) and marrow reticulin fibrosis.

\section{Result}

Total of 25 cases were studied of which $13(52 \%)$ were males, $12(48 \%)$ were females, $8(32 \%)$ were primary myelofibrosis and $17(68 \%)$ were secondary myelofibrosis. $13(52 \%)$ of cases aged between 41-60 years. Among primary myelofibrosis $6(75 \%)$ were males and $2(25 \%)$ were females, $75 \%$ (6) presented with pancytopenia and had Hemoglobin between 4.1-6 gm/dL. All had thrombocytopenia but none had severe thrombocytopenia and Bleeding. 5(62.5\%) showed Hypercellular marrow, $5(62.5 \%)$ showed grade 2 fibrosis and 3(37.5\%) showed grade 3 fibrosis. 3(37.5\%) showed grade 1, 3(37.5\%) had grade 2, and $2(25 \%)$ had grade 3 Mean vessel density.4(50\%) had marked splenomegaly.

Among Secondary Myelofibrosis 11(64.71\%) were of Hematological Malignancies which includes AML(3),CML(2), $\quad$ CLL(1), $\quad$ MDS(1), $\quad$ NHL(3), $\mathrm{MM}(1) .7(41.1 \%)$ showed increased bone marrow cellularity which included $\operatorname{AML}(3), \operatorname{CML}(2), \operatorname{CLL}(1)$, NHL(1).15(88.2\%) showed grade 1 fibrosis with single case of CML and TB with grade 2 fibrosis.4(23.5\%) showed grade 1 Mean vessel density which includes AML(2) and CML(2).4(23.5\%) showed mild splenomegaly which includes AML(3), Metastatic Deposit from Breast carcinoma(1) and 2(11.7\%) cases of CML showed marked splenic enlargement.

\section{Discussion}

Bone marrow fibrosis can be idiopathic or secondary which occurs secondary to release of fibroblast growth factor ${ }^{[10-12]}$. Fibroblast growth factors include PDGF, Epidermal growth factor,Endothelial growth factor,TGF -beta. These factors are present in the alpha -granules of megakaryocytes. Bone marrow fibrosis can also result from stimulation of fibroblasts secondary to release of tumor necrosis factor alpha, IL-1alpha and IL -1beta which are produced by marrow cells. According to Barosi.G et al (2003) ${ }^{[13]}$ and ozen.s et al (1997) ${ }^{[14]}$, idiopathic myelofibrosis characteristically occurs after 50 years of age. And the mean age at diagnosis is 65years. In this study most of cases of primary myelofibrosis are over 50years and the mean age group at diagnosis is 54 . According to okamura.T et al (2001) ${ }^{[15]}$ myelofibrosis, in adults occurs with about 
Table 1 : Correlation of MVD with Reticulin Fibrosis and Splenomegaly In PMF.

\begin{tabular}{|c|c|c|c|c|c|}
\hline S.NO & AGE/SEX & $\begin{array}{c}\text { BONE MARROW } \\
\text { CELLULARITY }\end{array}$ & $\begin{array}{c}\text { RETICULIN } \\
\text { FIBROSIS }\end{array}$ & MVD \\
\hline 1 & $17 / \mathrm{M}$ & Hypercellular & Grade II & Grade III & Marked \\
\hline 2 & $72 / \mathrm{M}$ & Hypercellular & Grade II & Grade II & Mild \\
\hline 3 & $56 / \mathrm{M}$ & Hypocellular & Grade III & Grade I & Marked \\
\hline 4 & $52 / \mathrm{F}$ & Hypercellular & Grade II & Grade III & Moderate \\
\hline 5 & $65 / \mathrm{M}$ & Hypercellular & Grade II & Grade II II & Moderate \\
\hline 6 & $58 / \mathrm{F}$ & Hypercellular & Grade II & Grade I & Marked \\
\hline 7 & $51 / \mathrm{M}$ & Hypocellular & Grade III & Grade I & Marked \\
\hline 8 & $60 / \mathrm{M}$ & Hypocellular & Grade III & & Ged \\
\hline
\end{tabular}

Table 2 : Correlation of MVD with Reticulin Fibrosis And Splenomegaly in Secondary Myelofibrosis.

\begin{tabular}{|c|c|c|c|c|c|c|}
\hline S.NO & AGE/SEX & DIAGNOSIS & $\begin{array}{l}\text { BONE MARROW } \\
\text { CELLULARITY }\end{array}$ & $\begin{array}{l}\text { RETICULIN } \\
\text { FIBROSIS }\end{array}$ & MVD & $\begin{array}{c}\text { SPLENIC } \\
\text { ENLARGEMENT }\end{array}$ \\
\hline 1 & $40 / F$ & AML & Hypercellular & Grade I & Nil & Mild \\
\hline 2 & $33 / F$ & SLE & Normocellular & Grade I & Nil & Nil \\
\hline 3 & $60 / \mathrm{M}$ & AML & Hypercellular & Grade I & Grade I & Mild \\
\hline 4 & $55 / F$ & SCC - TONSIL & Hypocellular & Grade I & Nil & Nil \\
\hline 5 & $75 / F$ & AML & Hypercellular & Grade I & Grade I & Mild \\
\hline 6 & $15 / M$ & $\mathrm{~TB}$ & Normocellular & Grade I & Nil & Nil \\
\hline 7 & $33 / F$ & $\mathrm{CML}$ & Hypercellular & Grade II & Grade I & Marked \\
\hline 8 & $46 / \mathrm{M}$ & $\mathrm{CML}$ & Hypercellular & Grade I & Grade I & Marked \\
\hline 9 & $77 / F$ & CLL & Hypercellular & Grade I & Nil & $\mathrm{Nil}$ \\
\hline 10 & $42 / \mathrm{M}$ & $\mathrm{TB}$ & Normocellular & Grade II & Nil & Nil \\
\hline 11 & $54 / F$ & MDS & Hypocellular & Grade I & Nil & Nil \\
\hline 12 & $65 / M$ & $\mathrm{NHL}$ & Normocellular & Grade I & Nil & Nil \\
\hline 13 & $42 / F$ & $\begin{array}{c}\text { METASTATIC } \\
\text { DEPOSIT - BREAST }\end{array}$ & Normocellular & Grade I & Nil & Mild \\
\hline 14 & $48 / \mathrm{M}$ & NHL & Hypercellular & Grade I & Nil & Nil \\
\hline 15 & $44 / F$ & $\begin{array}{c}\text { METASTATIC } \\
\text { DEPOSIT - BREAST }\end{array}$ & Normocellular & Grade I & Nil & Nil \\
\hline 16 & $50 / F$ & $\mathrm{NHL}$ & Normocellular & Grade I & Nil & Nil \\
\hline 17 & $70 / \mathrm{M}$ & MM & Normocellular & Grade I & Nil & Nil \\
\hline
\end{tabular}




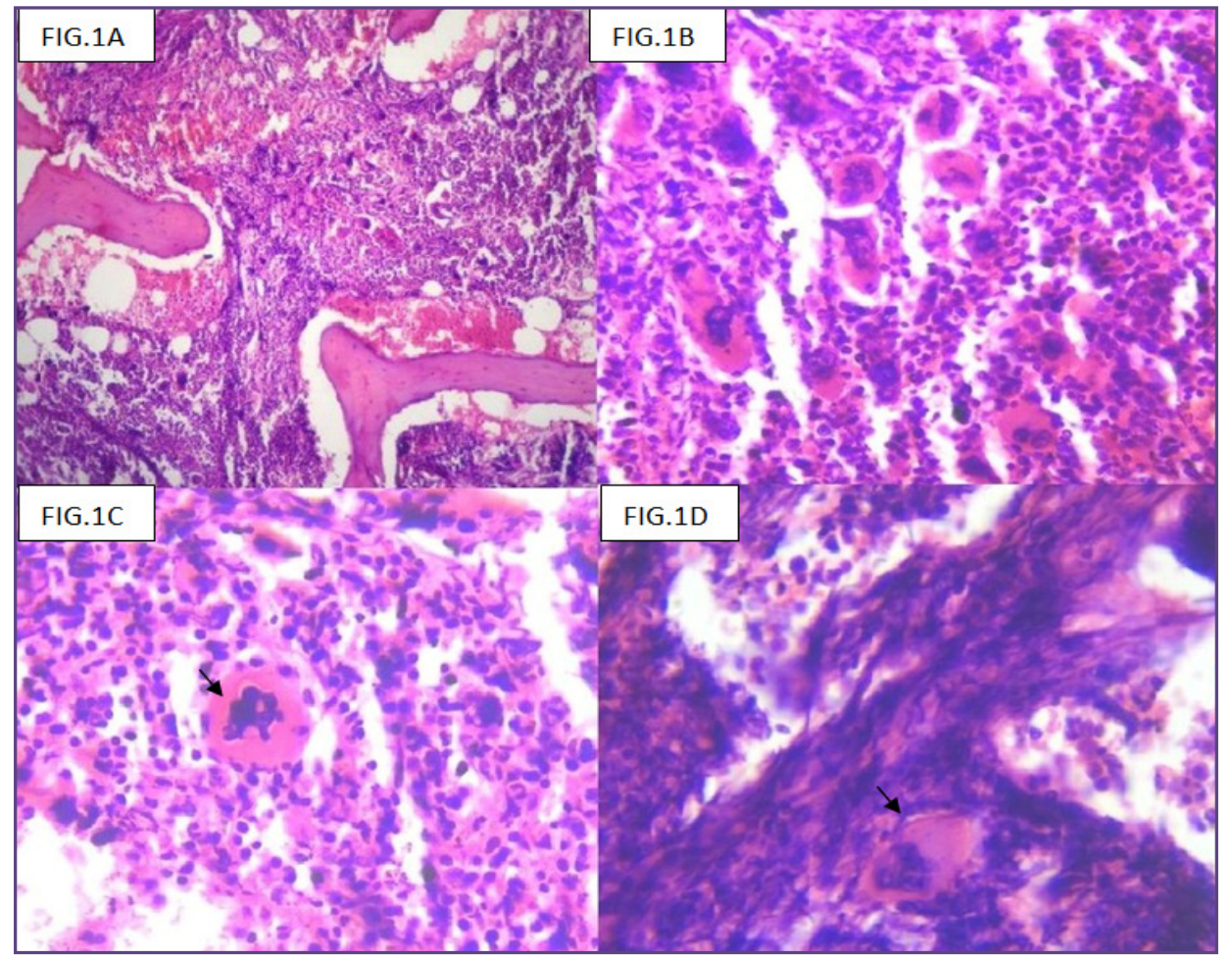

FIG. [1A-1D].1A. PMF With Trilineage Hyperplasia, 1B. PMF with Megakaryocytic Clustering, 1C. Abnormally lobated megakaryocyte, 1D. Entrapped megakaryocyte within fibrosis [H\&E STAIN, 40X].

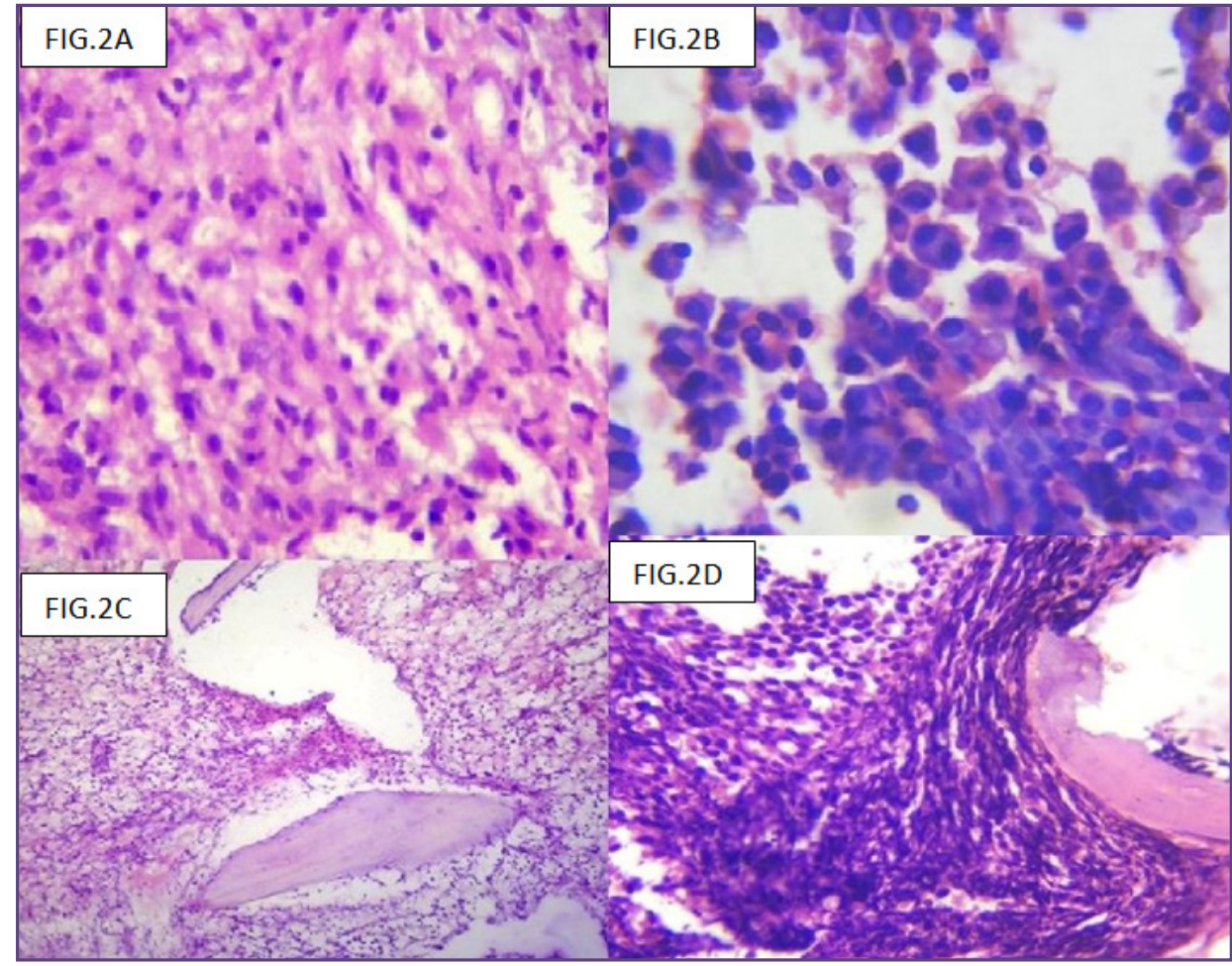

FIG. [2A-2D].2A. Epithelioid Cell Granuloma 2B. Sheets of plasma cells in MM, 2C. Radiation induced fibrosis in SCC, 2D. CLL with fibrosis [H\&E STAIN, 40X]. 


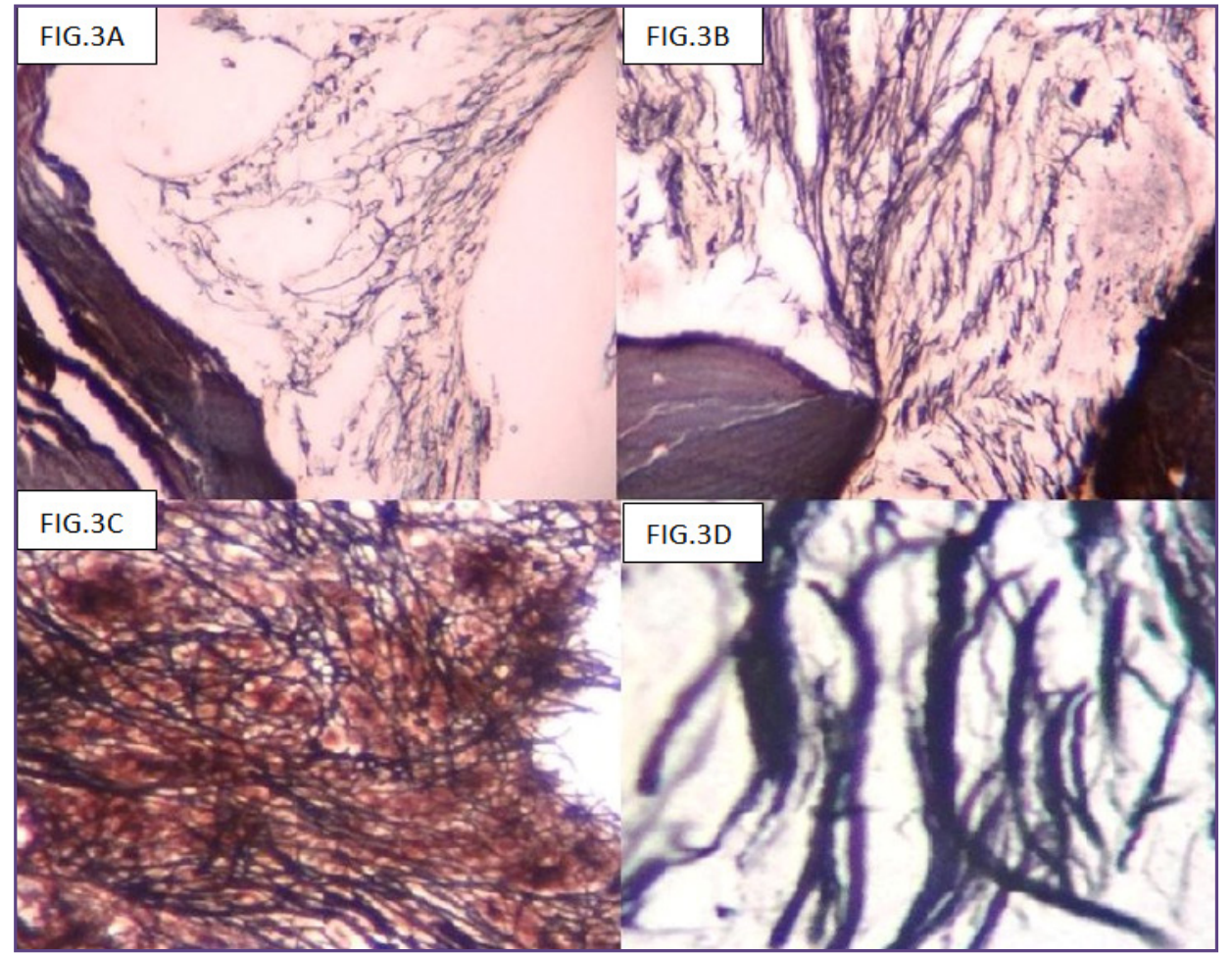

FIG. [3A-3D], 3A. Fibrosis Grade I, 3B. Firosis Grade II, 3C. Fibrosis Grade III, 3D. Fibrosis Grade III [Reticulin Stain - 3A,3B :4X, 3C:10X, 3D:40X ].

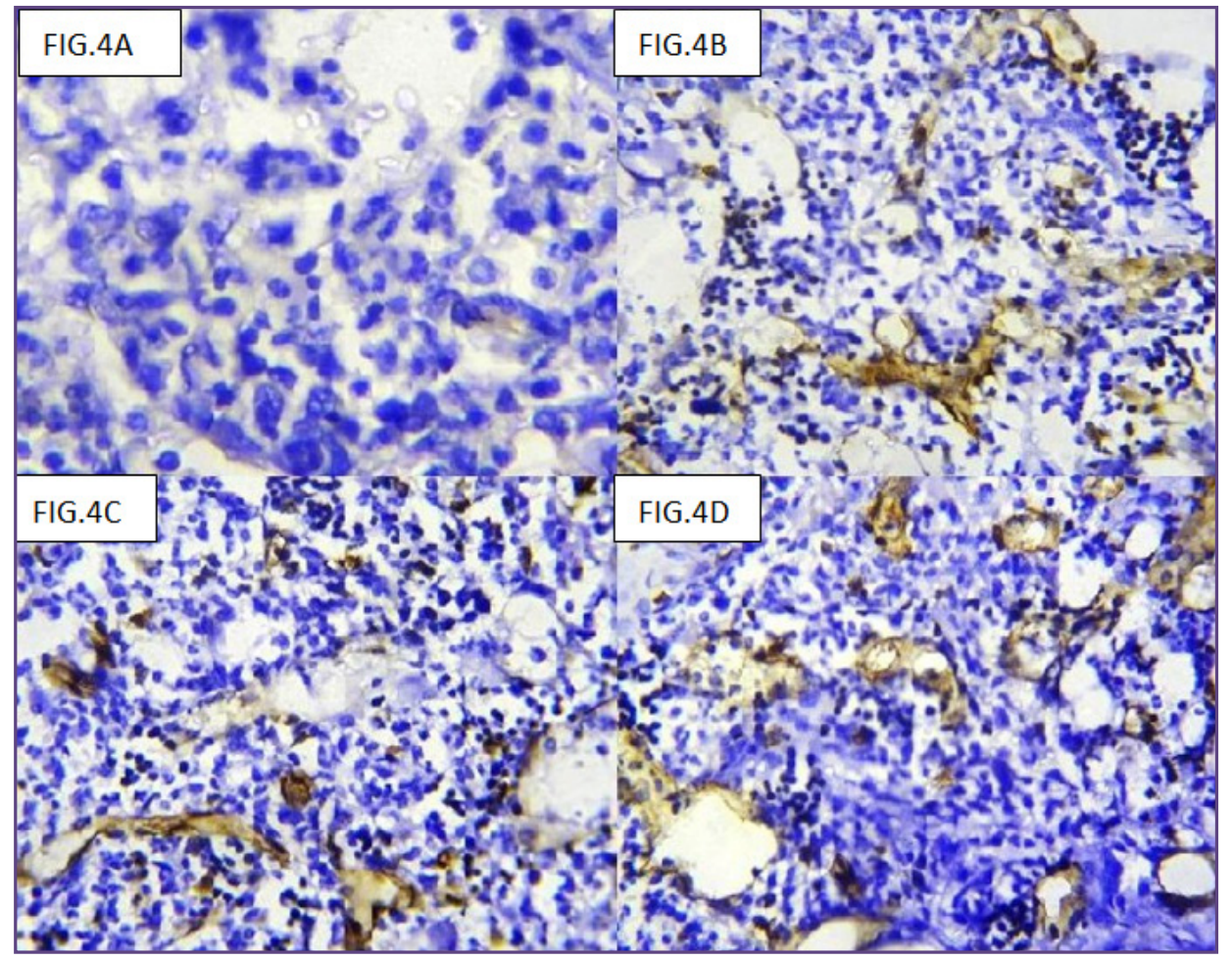

FIG. [4A-4D], 4A. No increase in MVD, 4B. MVD GRADE I, 4C. MVD GRADE II, 4D. MVD GRADE III [CD 34 STAIN, 10X ]. 
equal frequency in men and women. In this study male to female is $3: 1$ (male preponderance). This may be probably due to low number of cases involved in the study. In addition to this in this study ratio of primary and secondary myelofibrosis is found to be $1: 2(1: 2)$ which indicates that secondary myelofibrosis is more common than primary.

Among 8 of primary myelofibrosis cases, all the cases showed reticulin fibrosis. Reticulin fibrosis was patchy in the hypercellular marrow and diffuse in case of hypocellular marrow. Hence it can be considered that increase in reticulin fibrosis is associated with decrease in the bone marrow cellularity. This is similar to results given by Wolf and Nieman ${ }^{[16]}$. Out of 8 cases, 3 cases showed megakaryocytic clustering and 5 cases showed dysplastic megakaryocytes. Intrasinusoidal hematopoiesis was observed in 2 cases. According to Wolf and Nieman, intrasinusoidal hematopoiesis was apparent in the hypocellular marrows. But in this study intrasinusoidal hematopoiesis was readily observed in cellular marrows with accompanying fibrosis. According to Mesa et al and panteli et al ${ }^{[17]}$ angiogenesis is more evident in Primary myelofibrosis. In their study patients with PMF were found to have significantly higher values of MVD than those with other disorders. In this study also there is a substantial increase in the mean vascular density in case of PMF when compared to other cases. Although only some cases (3 out of 8 cases) showed intense increase in the mean vascular density. This may be due to decrease in the bone marrow cellularity with accompanying increase in the reticulin fibrosis. According to Varki et $\mathrm{al}^{[18]}$ and Silverstein et al $90 \%$ of patient had splenomegaly. In this study (7 out of 8 ) $87.5 \%$ of patients had splenomegaly. Among these patients, one underwent splenectomy due to complicating symptoms of splenomegaly. Histopathological examination of splenectomy specimen revealed extramedullary hematopoiesis. Increase in the spleen size is irrespective of degree of fibrosis. Some of the patient with mild degree of fibrosis had more splenomegaly and some patients with high degree of reticulin fibrosis had mild splenomegaly. This reflects the fact that splenomegaly does not depends upon the degree of reticulin fibrosis. This is comparable to study results given by BC Wolf and RS Nieman. Some patients had increase in the spleen size without increase in the Mean vascular density. In such cases, increase in spleen size may be attributed to the increased disease duration.

\section{Conclusion}

Bone marrow fibrosis is not Unique to Primary Myelofibrosis. It occurs in variety of Hematological and Non Hematological disorders. Primary Myelofibrosisis usually associated with increase in mean vascular density. Increase in Bone marrow cellularity is accompanied by increase in MVD and Increase in Fibrosis is accompanied by Decreased cellularity. Increase in mean vascular density, megakaryocytic clustering and intrasinusoidal haematopoiesis are considered to be the pre-existing events leading to leading to reticulin fibrosis and splenomegaly. Hence studying the Mean vascular density and correlating MVD with fibrosis, splenomegaly will serve as important factor in predicting Disease progression and patient survival.

\section{Abbreviations}

CD-Cluster of Differentiation, MVG- Microvessel Density Grading, MVD-Microvessel Density, AML-Acute Myeloid Leukemia, CML- Chronic Myelogenous Leukemia, CLL Chronic Lymphocytic Leukemia, MDS - Myelodysplastic Syndrome, NHL-Non Hodgkins Lymphoma, MMMultiple Myeloma, PDGF-Platelet derived growth Factor, TGF-Transforming Growth Factor,IL-Interleukin,PMFPrimary Myelofibrosis,TB-Tuberculosis,SLE-Systemic Lupus Erythematosis, SCC- Squamous Cell Carcinoma.

\section{Acknowledgements}

I take this Opportunity to express my sincere thanks and gratitude to our beloved Professor Dr. M.MURTHY. M.D., Head of the Department of Pathology, Coimbatore Medical College for his Guidance and My family members for their constant Support.

\section{Reference}

1. 1. Hotta $\mathrm{T}$, Utsumi $\mathrm{M}$, Katoh $\mathrm{T}$, et al: Granulocytic and stromal progenitors in the bone marrow of patient with primary myelofibrosis. Scand J Haematol 34:251,1985.

2. 2. Folkman J, Seminars in Medicine of the Beth Isreal Hospital, Boston: Clinical applications of research on angiogenesis. N Engl J Med.1995;333:1757-1763.

3. 3.Perez-Atayde AR, Sallan SE, Tedrow U, Connors S, Allred E, Folkman J: Spectrum of tumor angiogenesis in the bone marrow of children with acute lymphoblastic leukemia. Am J Pathol. 1997; 150:815-821.

4. 4.Hussong JW, Rodgers GM, Shami PJ: Evidence of increased angiogenesis in patients with acute myeloid leukemia. Blood. 2000;95:309-313.

5. 5.Pruneri G, Bertolini F, Soligo D, et al: Angiogenesis in myelodysplastic syndromes. Br J Cancer. 1999;81:13981401.

6. 6.Aguayo A, Kantargian H, Talpaz M,et al: Increased angiogenesis in chronic myeloid leukemia and myelodysplastic syndromes(abstract).Blood. 1998;92(suppl 1):607a.

7. 7.Vacca A, Ribatti D, Presta M, et al: Bone marrow neovascularisation, plasma cell angiogenic potential, and 
matrix metalloproteinase-2 secretion parallel progression of human multiple myeloma. Blood. 1999;93:3064-73.

8. 8.Weidner, N. Tumor angiogenesis: Review of current applications in tumor prognostication. Seminars in Diagnostic Pathology,1993; 10, 302-313.

9. 9. Mesa, R.A., Hanson, C.A., Rajkumar, S.V., Schroeder, G. \& Tefferi, A. Evaluation and clinical correlation of bone marrow angiogenesis in myelofibrosis with myeloid metaplasia. Blood, 2000;96, 3374-80.

10. 10.Soini Y, Kamel D , Apaja-Sarkkinen M, et al : Tenascin immunoreactivity in normal and pathological bone marrow. J Clin Pathol 1993;46;218

11. 11. Reily JT, Nash JRG: Vitronectin (serum spreading factor): Its localization in normal and fibrotic tissue . J Clin Pathol 1988;41 : 1269

12. 12.Le Bousse-Kerdiles MC, Martyre MC, et al : Involement of the fibrogenic cytokines, TGF-b and bFGf, in the pathogenesis of idiopathic myelofibrosis. Pathol Biol $2001 ; 49: 153$.
13. 13.Barosi G: Myelofibrosis with myeloid metaplasia. Hematol Clin North Am 2003;17:1211.

14. 14. Ozen S, Ferhanoglu B, Senocak M, Tuzuner N: Idiopathic myelofibrosis (agnogenic myeloid metaplasia). Leuk Res 1997;21:125.

15. 15.Okamura T, Kinukawa N, Niho Y, Mizoguichi H: Primary chronic myelofibrosis: Clinical and prognostic evaluation in 336 japanese patients Int J Hematol 2001;73:194.

16. 16. Wolf BC, Neiman RS: Myelofibrosis with myeloid metaplasia: Pathophysiological implications between bone marrow changes and progression of splenomegaly. Blood 1985:65:803.

17. 17.Panteli, K., Zagorianakou, N.,Bai, M., Katsaraki, A., Agnantis, N.J., Bourantas, K. Angiogenesis in chronic myeloproliferative diseases detected by CD34 expression. European Journal of Haematology, 2004:72,410-415.

18. 18.Varki A, Lottenberg R, Griffin R, Reinhard E: The syndrome of idiopathic myelofibrosis: Clinicopathologic review with emphasis on the prognostic variables predicting survival. Medicine (Baltimore) 1983:62:353.

*Corresponding author:

Dr. Anbu Lenin Kulandaivel, No.24, Gandhi Road, S5, Third Floor, Visalakshi Apartments, Opposite Ganesan Hospital, Salem -636 007., Tamil Nadu, India. Phone: +91 9944243745

Email: dranbulenin@gmail.com

Financial or other Competing Interests: None. 